\title{
Renal Cell Carcinoma presenting as a Right Atrial Mass
}

\author{
${ }^{1}$ Arun Subramanian, ${ }^{2}$ Minati Choudhary, ${ }^{3}$ Ujjwal Chowdhary
}

\begin{abstract}
Renal cell carcinoma (RCC) has a tendency to invade the renal vein and thereby reach the right heart through inferior vena cava (IVC). This may necessitate a combined surgical procedure usually under cardiopulmonary bypass (CPB). In the following discussion, we shall present a case of right RCC extending into the right atrium. The patient underwent a radical nephrectomy followed by removal of the tumor from right atrium, IVC and hepatic vein under CPB.
\end{abstract}

Keywords: Renal cell carcinoma, Inferior vena cava, Right atrium, Transesophageal echocardiography.

How to cite this article: Subramanian A, Choudhary M, Chowdhary U. Renal Cell Carcinoma presenting as a Right Atrial Mass. J Perioper Echocardiogr 2014;2(2):65-67

\section{Source of support: Nil}

Conflict of interest: None

\section{CASE REPORT}

A 65-year-old, $70 \mathrm{~kg}$ gentleman was admitted with stage IV right renal cell carcinoma (RCC). Ultrasound examination of the abdomen revealed a large right renal mass measuring $21 \times 15.2 \mathrm{~cm}$ with extension of the tumor into the inferior vena cava (IVC). Transthoracic echocardiography (TTE) revealed a right atrial (RA) mass with a suspicious entry into the pulmonary artery. The computed tomographic imaging findings were complimentary to the ultrasonogram. The patient was scheduled for a right radical nephrectomy and removal of the RA mass under cardiopulmonary bypass (CPB). The anesthesia management and monitoring techniques were routine as per any other major cardiovascular procedure under $\mathrm{CPB}$ except the site of central venous pressure monitoring which was approached through the left internal jugular vein (IJV). An X Matrix iE 33 (Philips, Bothel, USA) transesophageal echocardiography (TEE) probe was inserted. A midesophageal four-chamber

\footnotetext{
${ }^{1}$ Senior Resident, ${ }^{2,3}$ Professor

1,2Department of Cardiac Anesthesia, All India Institute of Medical Sciences, New Delhi, India

${ }^{3}$ Department of Cardiac Surgery, All India Institute of Medical Sciences, New Delhi, India

Corresponding Author: Arun Subramanian, Senior Resident Department of Cardiac Anesthesia, All India Institute of Medical Sciences, New Delhi, India, Phone: 09968721687, e-mail: mkknso@gmail.com
}

revealed the tumor to be extending into the right atrium till the tricuspid valve (Fig. 1). The mass was heterogeneous and mobile; with a well defined irregular border and a continuation into IVC. There was no evidence of any tricuspid valve abnormality. The probe was advanced a little, turned right and rotated to $97^{\circ}$ till the tumor mass is seen in IVC-RA junction (Fig. 2). Advancement of the probe till the hepatic venous view showed the presence of extensive thrombus at IVC-hepatic vein junction (Fig. 3) as well as in hepatic vein (Fig. 4). The main pulmonary artery (MPA) as well as its right and left branches were seen to be free of any mass. A complete TEE examination was done for all the cardiac structure to exclude the presence of any thrombi, valve regurgitation, pericardial effusion or tamponade physiology. The ventricular function was normal. After the primary examination TEE was checked every few minutes during the dissection of the renal mass to monitor for the changes in thrombus position or for frank tumor embolization.

The patient underwent midline celiotomy, and resectability was determined, limiting dissection around IVC as much as possible. The laparotomy incision was extended cephalad with a median sternotomy. The apex of the tumor thrombus was palpated in the IVC and confirmed to extend till IVC-RA junction. Mild hypothermic $\left(32^{\circ}\right) \mathrm{CPB}$ using angled venous cannulas into superior vena cava, external iliac vein and ascending aorta was performed. After cardioplegic arrest, the first step was to dissect and clamp the MPA before tumor manipulation to prevent pulmonary artery thromboembolism. The next step was to isolate and control the cavoatrial junction above tumor using umbilical tape. IVC was opened below hepatic vein till the renal vein. With combined blunt and sharp dissection intervening tumor tissue was dissected till the atriocaval junction superiorly and venacavotomy inferiorly. The hepatic vein was freed of tumor tissue. A TEE examination revealed no remnant tumor bits. The patient was weaned from $\mathrm{CPB}$, the chest and the abdomen was closed in layers.

\section{DISCUSSION}

Primary neoplasms of the heart account for at least $40 \%$ of all cardiac tumors. ${ }^{1}$ On the other hand, metastatic carcinomas from abdomen and pelvis constitute the majority of the cardiac malignancies. Renal cell carcinoma 


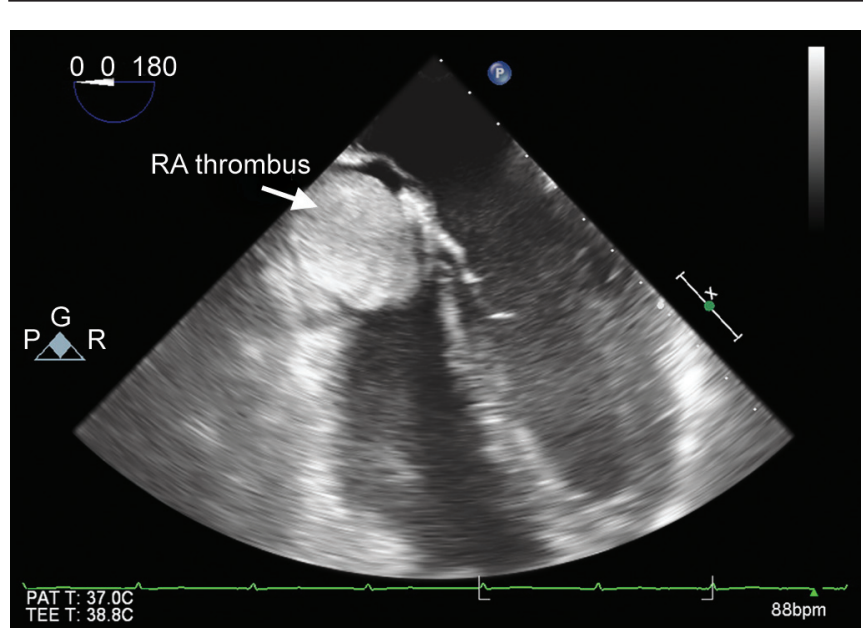

Fig. 1: Midesophageal four-chamber view demonstrating the tumor in the right atrium

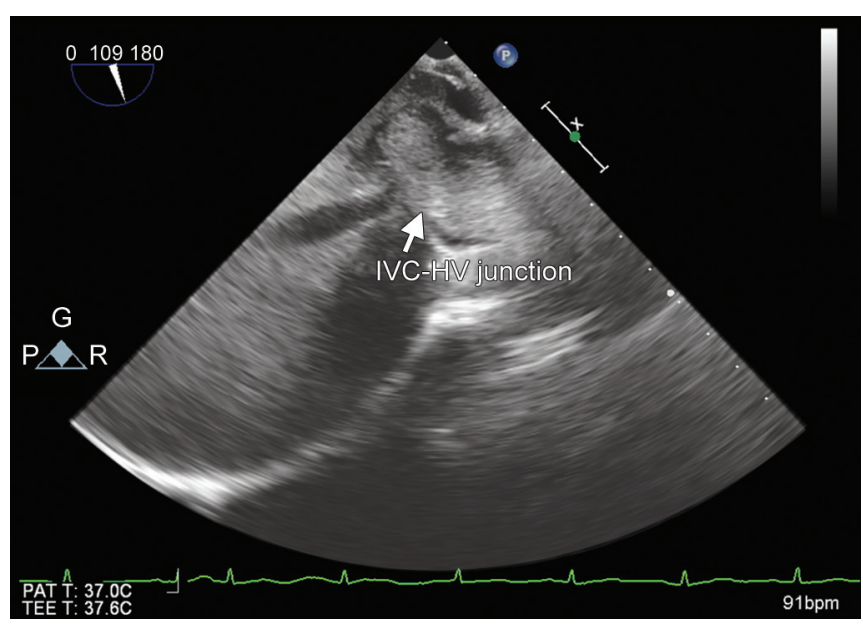

Fig. 3: Hepatic venous view, tumor at IVC-hepatic vein junction

is one such malignant tumor which has the propensity to invade the IVC and extend into the right heart. ${ }^{2}$ Tumor associated thrombus, hemorrhage and calcification can be misinterpreted as intracardiac vegetations or mural thrombi when small in sizes. Echocardiographically thrombi appear as masses, usually sessile, pedunculated, smooth walled and show soft tissue reflectance. It is usually visible throughout the cardiac cycle. At times they may be echo free, heterogeneous and have areas of calcification. Vegetations on the other hand usually developed on the low pressure zone (side of a valve). It has chaotic motion, independent of cardiac cycle and show soft tissue reflectance. ${ }^{3}$

Renal cell carcinoma is known to be the most lethal of all the genitourinary neoplasms. The most common factors influencing prognosis are local infiltration to perinephric tissue, lymph node involvement and distant metastasis and involvement of IVC and right atrium irrespective of the level of tumor extension. Radical or partial nephrectomy remains the mainstay of treatment in cases of localized and locally advanced diseases. ${ }^{4}$

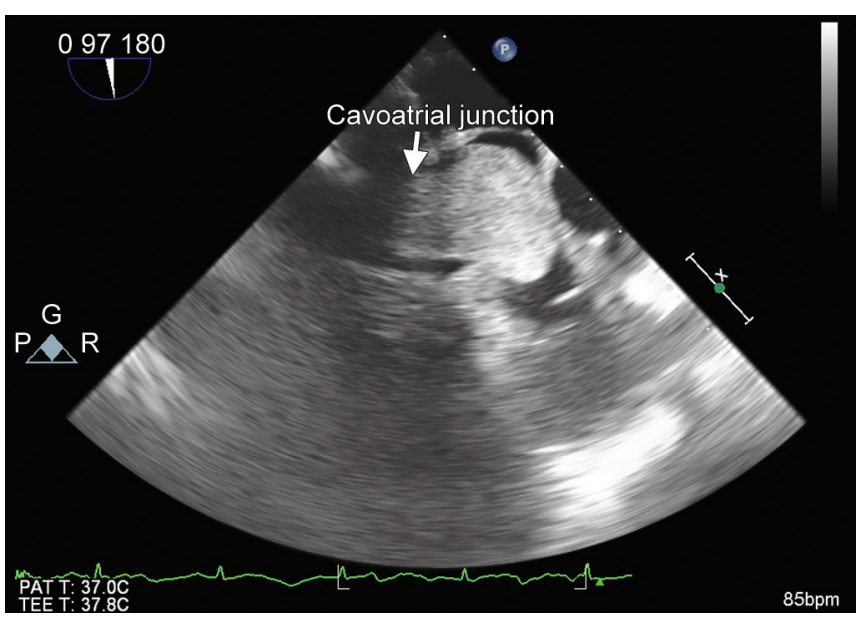

Fig. 2: Tumor at the right atrium-IVC junction, taken in advancement and turning of the probe $\left(97^{\circ}\right)$ from four-chamber view

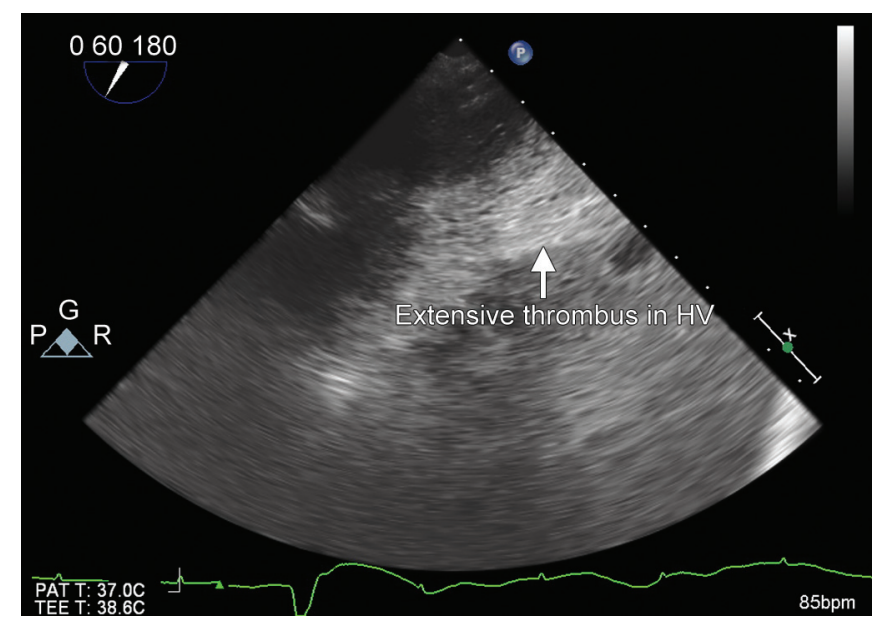

Fig. 4: Hepatic venous view tumor at hepatic vein

Temporary balloon occlusion of IVC as described by Yang $\mathrm{Y}$ et al is a simple and reliable technique that decreases the risk of pulmonary embolism and facilitates the resection of renal tumors with levels of II and III IVC thrombus. ${ }^{5}$ The surgical planning mainly depends upon the level of tumor thrombus. RCC with suprarenal (intrahepatic tumor thrombus) can be resected without CPB with control of IVC with clamp or tourniquet both above and below the tumor thrombus and around the renal vein to prevent tumor embolus. However, tumors with level III and IV IVC thrombus (retrohepatic and subdiaphragmatic) needs $\mathrm{CPB}$ for its complete removal as in our patient. ${ }^{6}$

The use of CPB can reduce the amount of blood loss, minimizes the chances of pulmonary embolism and ensure adequate removal of the tumor from RA and IVC. In some cases hypothermic circulatory arrest allows complete resection of the tumor in a virtually bloodless field albeit some systemic complications. ${ }^{7,8}$

Central venous cannulation can pose a serious challenge in these patients. The right IJV which is 
usually cannulated in cardiac surgeries can sometimes dislodge the RA mass. Alternatively the left IJV (as in our patient) or femoral vein can be accessed for central venous pressure monitoring in these patients. In case the femoral vein is employed, it has to be borne in mind that during the clamping of IVC for removal of the tumor the central venous pressure may be erroneous. Accordingly fluid management can be guided by the hemodynamic status of the patient and by TEE.

Noncardiac surgery has been increasingly identified as a potential valuable indication for TEE. ${ }^{9}$ We agreed with the practice guidelines by American Society of Anesthesiologists and Society of Cardiovascular Anesthesiologists that TEE can be a complementary tool for detection of tumor extension into the RA and IVC, apart from CT and MRI. It can act as an intraoperative tool for detection of any embolism, associated valvular pathology and septal defects which may necessitate additional surgical interventions.

Continuous TEE monitoring in our patient helped us to rule out the occurrence of any embolic event during intraoperative period..$^{10}$ Likewise TEE can be helpful at the time of separation from CPB to look for intracardiac air, myocardial function and residual tumor fragments. In our case the patient was successfully weaned off $\mathrm{CPB}$ without any complications.

\section{REFERENCES}

1. Bruke A, Virmani R. Tumors of heart and great vessels: Atlas of tumor pathology. 3rd series, fascicle 16. Washington DC; Armed Force Institute of Pathology; 1995. p. 171-177.

2. Allen G, Klingman R, Ferraris VA, Fisher H, Harte F, Singh A. Transesophageal echocardiography in the surgical management of renal cell carcinoma with intracardiac extension. J Cardiovasc Surg 1991 Nov-Dec;32(6):833-836.
3. Pepi M, Evangelista A, Nihoyannopoulos P, Flachskampf FA, Athanassopoulos G, Colonna P, Habib G, Ringelstein EB, Sicari R, Zamorano JL, et al. Recommendations for echocardiography use in the diagnosis and management of cardiac sources of embolism: European Association of Echocardiography (EAE) (a registered branch of the ESC). Eur J Echocardiogr 2010 Jul;11(6):461-476.

4. Blute ML, Leibovich BC, Lohse CM, Cheville JC, Zincke H. The Mayo Clinic experience with surgical management, complications and outcome for patients with renal cell carcinoma and venous tumour thrombus. BJU Int 2004 Jul;94(1): 33-41.

5. Yang Y, Sun S, Xiao X, Song Y, Cai W, Wang M, Zhang X. Temporary balloon occlusion of inferior vena cava in resection of renal tumor with vena cava thrombus extension. Urology 2009 Mar;73(3):645-648.

6. Wotkowicz C, Wszolek MF, Libertino JA. Resection of renal tumor invading the vena cava. Urol Clin North Am 2008 Nov; 35(4):657-671.

7. Skinner DG, Pritchett TR, Lieskovsky G, Boyd SD, Stiles QR. Vena cava involvement by renal cell carcinoma. Surgical resection provides meaningful long-term results. Ann Surg 1989 Sep;210(3):387-392.

8. Chowdhury UK, Mishra AK, Seth A, Dogra PN, Honnakere JH, Subramaniam GK, Malhotra A, Malhotra P, Makhija N, Venugopal P. Novel technique for tumor thrombectomy for renal cell carcinoma with intra-arterial tumor thrombus. Ann Thorac Surg 2007 May;83(5):1731-1736.

9. American Society of Anesthesiologists and Society of Cardiovascular Anesthesiologists Task Force on Transesophageal Echocardiography. Practice guidelines for perioperative transesophageal echocardiography. An updated report by the American Society of Anesthesiologists and the Society of Cardiovascular Anesthesiologists Task Force on Transesophageal Echocardiography. Anesthesiology 2010 May;112(5):1084-1096.

10. Sharma V, Cusimano RJ, McNama P, Wasowicz M, Ko R, Meineri $M$. Intraoperative migration of an inferior vena cava tumor detected by transesophageal echocardiography. Can J Anesth 2011 May;58(5):468-470. 